\title{
Drug therapies in type 2 diabetes: an era of personalised medicine
}

\author{
Authors: Tahseen A Chowdhury ${ }^{A}$ and Paul Grant ${ }^{B}$
}

Optimal management of diabetes involves a multidisciplinary approach. Prioritisation of lifestyle change, blood pressure and lipid control, and regular screening for complications are advocated in most international guidelines. Good glucose control, however, remains an important aim of treatment, although it is increasingly recognised that glucose targets should be individualised, with less stringent targets for older patients with significant comorbidities.

In recent years, a number of newer therapies for hyperglycaemia have become available. This review aims to discuss currently available options for patients with type 2 diabetes, and also discusses potential new therapies that may be on the horizon in the future.

KEYWORDS: Diabetes, hyperglycaemia, therapy

\section{Introduction}

The prevalence of diabetes is rising rapidly worldwide. At the present rate of increase, the number of people living with diabetes in the UK is predicted to rise to over 5 million by 2025 , with the predominant growth in type 2 diabetes (T2D). ${ }^{1}$ Diabetes contributes to significant morbidity and mortality from micro- and macrovascular complications, and is responsible for heavy financial and societal costs - mostly from treatment of complications.

International guidelines for the management of diabetes highlight the paramount importance of lifestyle change (improved diet, physical activity and smoking cessation) in controlling cardiovascular risk factors. ${ }^{2-4}$ Recently published National Institute for Health and Care Excellence (NICE) guidelines stress the need for lifestyle change, smoking cessation, blood pressure reduction and lipid lowering to reduce the risk of diabetes complications, particularly cardiovascular complications. ${ }^{2}$

A further important aspect of care for people with diabetes is screening for complications. In the UK, National Diabetes Audits have consistently shown that almost half of patients with

Authors: A consultant in diabetes, The Royal London Hospital, London, UK; ${ }^{\mathrm{B}}$ consultant in diabetes and endocrinology, Oxford Centre for Diabetes, Endocrinology and Metabolism, Oxford, UK diabetes do not undergo their key processes of care each year (weight, blood pressure, cholesterol, urine albumin creatinine ratio, serum creatinine, foot check, glucose control, eye screening, smoking advice) ${ }^{5}$

Glucose control remains important, especially in helping reduce the risk of microvascular complications (retinopathy, nephropathy and neuropathy). It is increasingly recognised in various guidelines that targets for glucose control need to be individualised. ${ }^{2-4}$ Thus, a glucose target for a recently diagnosed younger person with few comorbidities may be significantly lower compared with that of an older person with a long duration of diabetes and multiple comorbidities (Box 1). Drug therapy for diabetes has evolved over the last decade, such that newer agents enable clinicians to tailor therapy according to patients' needs and preferences. NICE guidelines suggest a clear pathway for glucose therapy (Fig 1). This article aims to describe the place for old and new drug therapies in the management of T2D, and to highlight some potential new therapies that may be available in the near future.

\section{Oral hypoglycaemic therapies}

\section{Metformin}

Mechanism of action

The main effect of metformin is to acutely decrease hepatic glucose production, mostly through inhibition of the mitochondrial respiratory chain complex $1 .^{6}$ The resulting decrease in hepatic energy status activates the AMP-activated protein kinase (AMPK) - a cellular metabolic sensor,

\section{Box 1. What factors should be considered when} deciding on glycaemic target?

Age/life expectancy/comorbidity

Duration of diabetes

Motivation, capacity for self-care, social support

Perhaps aim for tighter control in:

$>$ younger patients $(<65$ years)

shorter duration of diabetes ( $<10$ years)

few comorbidities (especially renal/cardiovascular)

$>$ higher motivation/knowledge 
Fig 1. NICE 2015 therapeutic pathway for glycaemic management of type 2 diabetes. BMI = body mass index; DPP-4i = dipeptidylpeptidase-4 inhibitors; GLP-1 = glucagon-like peptide-1 analogue; $\mathrm{MR}=$ modified release; $\mathrm{NPH}=$ neutral protamine Hagedorn; Pio = pioglitazone; SGLT-2i $=$ sodium glucose transporter-2 inhibitor; $\mathrm{SR}=$ standard release; $\mathrm{SU}=$ sulfonylurea

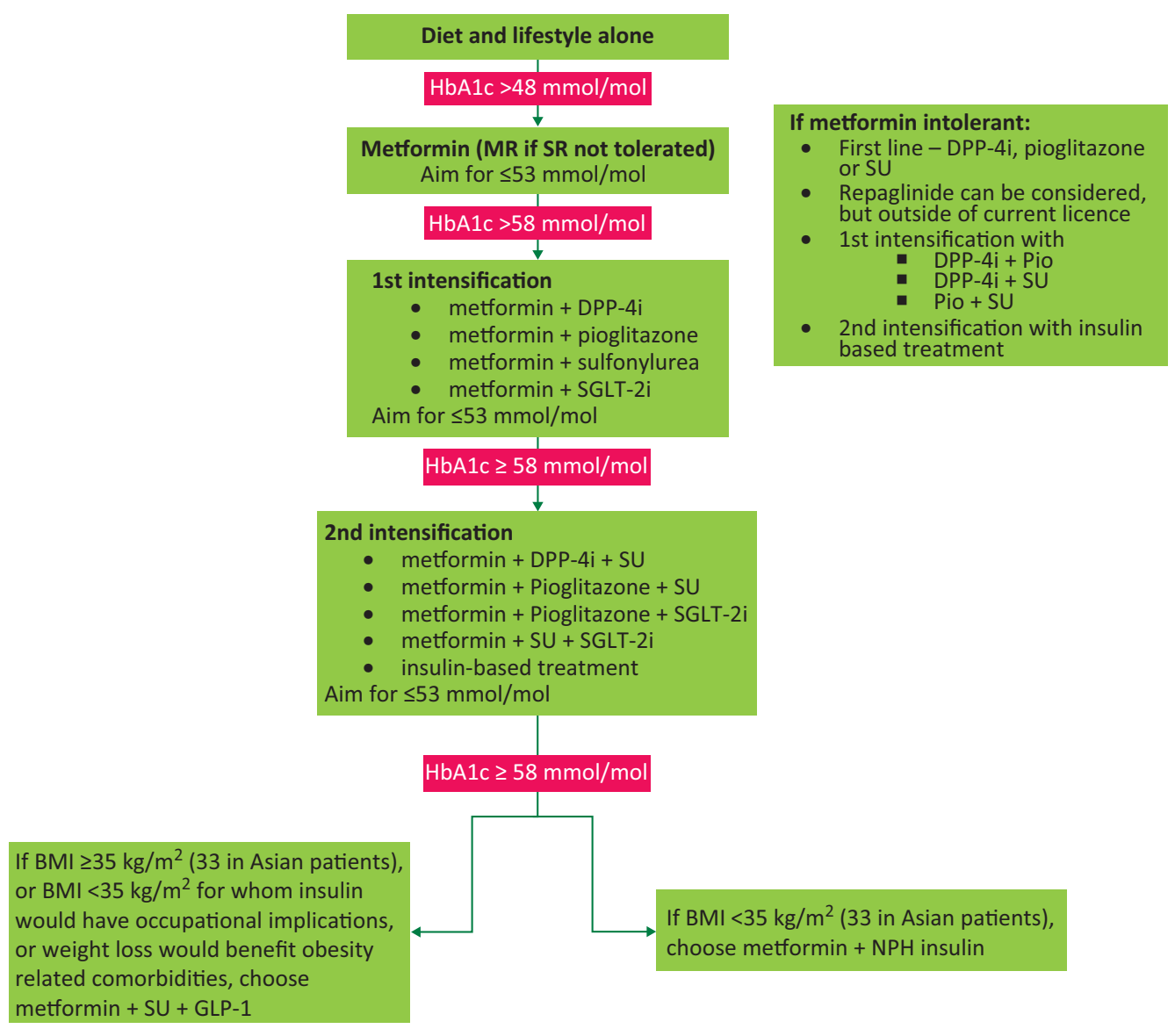

suppressing hepatic gluconeogenesis. Overall, this leads to improved insulin sensitivity and improved glucose uptake. There is evidence to suggest that the effect of metformin may also be mediated through acting on the gastrointestinal tract, possibly by increasing glucagon-like peptide-1 (GLP-1) levels. ${ }^{7}$

When should it be used?

Metformin has been shown to have a beneficial effect on mortality and cardiovascular disease in obese patients with T2D. ${ }^{8}$ As a result, it is deemed first line therapy in most patients with T2D. Metformin can be continued in conjunction with all other oral and injectable therapies. Use of metformin with insulin is desirable in patients with $\mathrm{T} 2 \mathrm{D}$, as it leads to reduced weight gain and lower insulin dosage. ${ }^{9}$ Metformin is safe in stable heart failure and should only be discontinued in patients with severe progressive renal impairment. ${ }^{10}$

\section{Typical dose}

In order to reduce the risk of gastrointestinal side effects, the starting dose should be $500 \mathrm{mg}$ once daily after main meal, gradually titrated to $1,000 \mathrm{mg}$ twice daily over the subsequent 4 weeks, always taken after meals. Metformin modified release can be used if side effects from standard metformin are prohibitive. Maximum effective dose is around $2.5 \mathrm{~g}$ daily.
Adverse effects and cautions

Diarrhoea, indigestion and nausea are common side effects of metformin, which can be mitigated by gradual dose increase and taking tablets after meals. Metformin should be stopped when estimated glomerular filtration rate (eGFR) is under $30 \mathrm{~mL} / \mathrm{min}$, and dosage should be reduced if eGFR is below $40 \mathrm{~mL} / \mathrm{min}$.

\section{Sulfonylureas}

Mechanism of action

Sulfonylureas are insulin secretagogues. They bind to the ATP-sensitive potassium channels on the pancreatic beta-cell to cause hyperpolarisation of the cell membrane, calcium channel opening and release of insulin. Their action therefore depends on the presence of residual pancreatic beta-cell function.

When should they be used?

They can be used first line when patients are not able to tolerate metformin or present with significant polyuria and polydipsia, as sulfonylureas can relieve osmotic symptoms quickly. They are often used as second line agents when control is inadequate with metformin alone. Sulfonylureas are effective in the treatment of many patients with monogenic diabetes (maturity onset diabetes of the young), which may affect as many as 70,000 people with diabetes in the UK. 
Typical dose

The commonest sulfonylurea used is gliclazide, with a typical starting dose of $40 \mathrm{mg}$ twice daily, titrated to a maximum of $160 \mathrm{mg}$ twice daily. Gliclazide can be given as a once daily modified release to aid concordance, dose range 30-120 mg daily. Glimepiride (Amaryl, Aventis Pharmaceuticals) is a once daily sulfonylurea, with a dose range of $1-6 \mathrm{mg}$ once daily.

\section{Adverse effects and cautions}

Sulfonylureas often cause weight gain, and should be used with caution in overweight or obese patients. The risk of significant hypoglycaemic events is increased in people with erratic eating habits, alcohol excess, or very tight glycaemic control, especially in older patients or those with renal impairment, where dosage may need to be reduced. There is ongoing concern about the potential adverse cardiovascular effects of sulfonylureas, although no definitive evidence for this is available. ${ }^{11}$

\section{Meglitinides}

\section{Mechanism of action}

Meglitinides are prandial glucose regulators that also act on the sulfonylurea receptor. However, they are very short acting and tend to work in a glucose-dependent manner. Thus, if prevailing glucose is high, they will stimulate insulin, and if low, they will cause less insulin stimulation and, in theory, less hypoglycaemia and weight gain compared with standard sulfonylureas.

When should they be used?

They are taken with food and, hence, if food times are erratic, they may aid meal time flexibility. ${ }^{12}$ They may also be useful in Muslim patients who wish to fast during Ramadan. ${ }^{13}$ Repaglinide has been suggested by recent NICE guidelines as being an unlicensed first line option for patients intolerant of metformin. The fact that it needs to be taken multiple times a day may inhibit concordance. It may be used in addition to metformin as a second line agent.

Typical dose

Repaglinide can be started at $0.5 \mathrm{mg}$ with meals, up to a maximum of $4 \mathrm{mg}$ per meal.

\section{Adverse effects and cautions}

Similar to sulfonylureas, meglitinides may cause some modest weight gain and hypoglycaemia. They should be used in with caution in patients with moderate renal and hepatic impairment.

\section{Thiazolidinediones}

\section{Mechanism of action}

The currently only available thiazolidinedione is pioglitazone, which acts via the perioxisome proliferator activated receptor gamma (PPAR- $\gamma$ ) pathway. Stimulation of these receptors leads to significant enhancement of insulin sensitivity through activation of intracellular pathways involved in insulin sensitivity. The effect is slow, and pioglitazone takes around 3-4 months to exert its maximal effect.
When should they be used?

Pioglitazone can be used as monotherapy, if metformin is not tolerated or is contraindicated, and as dual or triple therapy with metformin, gliptins and sulfonylureas. In addition, pioglitazone is licensed for use with insulin.

\section{Typical dose}

Pioglitazone is given initially as $15-30 \mathrm{mg}$ and increased up to $45 \mathrm{mg}$ as necessary.

\section{Adverse effects and cautions}

The main adverse effect of pioglitazone is fluid retention, and it is therefore absolutely contraindicated in people with known heart failure. Liver function test monitoring is required although there is some suggestion that pioglitazone may be beneficial in patients with non-alcoholic steatohepatitis (NASH). ${ }^{14}$ Weight gain of 3-5 kg may be seen with pioglitazone.

Through its action on bone marrow cells, pioglitazone can also cause a decrease in red blood cell production, with consequential mild anaemia. It has also been shown to increase the risk of distal fractures in postmenopausal women and, hence, is not recommended in women at high risk of fracture. ${ }^{15}$ An association with bladder cancer has been recently suggested, although absolute risk is small. ${ }^{16}$ Patients with a previous history of bladder cancer should not be prescribed pioglitazone.

\section{Dipeptidylpeptidase-4 inhibitors}

\section{Mechanism of action}

The incretin pathway appears to be an important regulator of glucose metabolism. The main incretin hormone is GLP-1, the levels of which are reduced in T2D. GLP-1 is rapidly degraded by the enzyme dipeptidylpeptidase-4 (DPP-4). Inhibition of DPP-4 leads to elevation of endogenous GLP-1 and enhanced insulin secretion. Gliptins are a class of oral hypoglycaemic agent that inhibit DPP-4, therefore enhancing and prolonging the physiological actions of endogenous incretin hormones.

When should they be used? Gliptins may be used first line where metformin is contraindicated or not tolerated. NICE guidelines now suggest they are a second or third line option, and may be used in addition to insulin. Their weight neutral effect makes them useful in overweight or obese patients.

\section{Typical dose}

Sitagliptin is generally started at $100 \mathrm{mg}$ once daily. Linagliptin is useful in patients with renal impairment because the dose does not need to be adjusted. It is used as $5 \mathrm{mg}$ once daily throughout the range of renal function, even down to chronic kidney disease (CKD) stage 5.

\section{Adverse effects and cautions}

Most studies have found no significant adverse effects for DPP-4 inhibitors. In general, gliptins appear to be safe and well tolerated, with few reported side effects - cold/flu-like symptoms, headache and dizziness. Pancreatitis has been 
reported as a risk with gliptins, although this increased risk appears small. ${ }^{17}$ Nevertheless, prescribers should be aware of the potential for pancreatitis in patients given gliptins.

\section{Alpha-glucosidase inhibitors}

Mechanism of action

Alpha-glucosidases break down complex carbohydrates in the small intestine for absorption into the bloodstream. Acarbose inhibits this enzyme resulting in a delay in digestion of carbohydrates and a relative decrease in blood glucose levels, especially post-prandially.

When should they be used?

Acarbose is rarely effective on its own and is limited to those who are unable to tolerate other glucose lowering medications. Acarbose is contraindicated in severe renal failure as well as hepatic failure. It does not appear in the latest NICE guidance, but is used in some circumstances, particularly in patients showing intolerance of other agents. Acarbose is rarely used in the UK, although it is more widely used in South Asia.

Typical dose

The initial dose is $50 \mathrm{mg}$ daily with food. This can be increased over a period of weeks to a maximum of $200 \mathrm{mg}$ three times daily, with meals. Side effects are dose-related, which can inhibit optimal doses.

\section{Adverse effects and cautions}

The reduced digestion of carbohydrates results in a predominance of gastrointestinal side effects, including bloating, flatulence, diarrhoea and abdominal pain. This often leads to poor concordance. Acarbose should be avoided in people with bowel problems. In patients treated with acarbose, hypoglycaemia induced by drugs used in addition to acarbose may not respond to sucrose because of the mechanism of action; therefore, patients need to be aware that hypoglycaemia needs to be treated with glucose.

\section{Sodium glucose transporter-2 inhibitors}

\section{Mechanism of action}

Sodium glucose transporter-2 (SGLT-2) is a transporter responsible for glucose reabsorption in the proximal convoluted tubule of the kidney. Blocking this transporter leads to glycosuria. SGLT-2 inhibitors (gliflozins) use this mechanism of action to induce renal glucose loss and, hence, lower plasma glucose and potentially lead to weight loss.

When should they be used?

These therapies have been subject to NICE technical appraisal and can be used second or third line, or with insulin. They have the benefit of improving glucose control and inducing modest weight loss of 1-4 kg; they are therefore useful in overweight or obese patients. A 2015 study of the use of empagliflozin in high-risk patients with diabetes and cardiovascular disease showed a $38 \%$ reduction in risk of cardiovascular death, 35\% reduction in risk of heart failure and 32\% reduction in all-cause mortality over 4 years. ${ }^{18}$ Numbers needed to treat to prevent one cardiovascular death over 3 years was 39 , which compares favourably to statins and angiotensin converting enzyme (ACE) inhibitors. These data need to be reproduced in other studies before gliflozins can be more widely advocated.

Typical dose

Dapagliflozin is given at a dose of 5-10 mg daily, canagliflozin $100-300 \mathrm{mg}$ once daily and empagliflozin $10-25 \mathrm{mg}$ daily.

\section{Adverse effects and cautions}

Predictably, inducing glycosuria may increase risk of urinary tract infection (UTI) or genital candidiasis, and this adverse effect occurs in around $8 \%$ of treated patients. Treatment with antibiotics or antifungals usually cures the problem and recurrent infections are uncommon. However, the drug should be avoided in patients with a history of recurrent or complicated UTI. The diuretic and hyponatraemic effect of thiazides may be enhanced by SGLT-2 inhibitors, and the two should not be combined. These agents are not licensed for use in significant renal impairment (eGFR under $45 \mathrm{~mL} / \mathrm{min}$ ).

Rare cases of euglycaemic ketoacidosis have been reported. ${ }^{19}$ These have been predominantly in patients with type 1 diabetes (T1D), in whom these agents are not licensed. Patients on these agents, however, should be warned that if they become acutely unwell with vomiting or abdominal pain, then a plasma or urine ketone level should be checked and, if elevated, the patient should be assessed in hospital.

\section{Injectable therapies}

\section{GLP-1 analogues}

\section{Mechanism of action}

GLP-1 is an 'incretin' hormone that acts to lower blood glucose levels by way of stimulating insulin release and blocking the production of glucagon. It is also recognised to act directly on the gut to slow down gastric secretions and motility and this has an impact on both the absorption of carbohydrates from the gut and patient satiety. ${ }^{20}$ They also have a mild weight loss effect, which is beneficial in the context of T2D. GLP-1 analogues include exenatide, liraglutide, lixisenatide and dulaglutide, and are administered as a regular subcutaneous injection.

\section{When should they be used?}

NICE guidelines suggest the indication for GLP-1 analogues is generally when maximal oral hypoglycaemic therapy has failed to bring the patient's HbAlc down to below $58 \mathrm{mmol} / \mathrm{mol}(7.5 \%)$ and the patient's body mass index (BMI) is greater than $35 \mathrm{~kg} / \mathrm{m}^{2}$. NICE also suggests that these agents may be used at a lower BMI where insulin therapy may have adverse impacts on occupation (such as driving), or where weight loss may benefit obesity-related comorbidities (such as obstructive sleep apnoea). They can also be considered for use as an insulin sparing agent in those patients already on insulin therapy especially when the insulin is contributing to weight gain, although this has to be done under a consultant-supervised multidisciplinary team.

\section{Typical dose}

GLP-1 analogues are prescribed as twice daily, once daily or once weekly injections depending on the product used. 


\section{Adverse effects}

Given that GLP-1 agonists primarily work via gastrointestinal mechanisms, they are not advised to be prescribed for those people with severe gastrointestinal disease. The major side effect of this class of drugs is gastrointestinal upset, ranging from mild nausea and bloating to severe vomiting or diarrhoea. Pancreatitis has been reported rarely, although should the patient develop acute severe abdominal pain with these drugs, pancreatitis should be considered.

\section{Insulin}

Insulin therapy has been reviewed previously in Clinical Medicine. ${ }^{21}$ Although patents on existing insulins have expired or are close to expiring in the next few years, there are no generic preparations available currently and, for biological drugs such as insulin, it can be difficult to produce an exact copy that will convincingly behave in the same way as the original insulin. A biological copy (known as a 'biosimilar') has small differences in the chemical structure compared with the original drug (despite being technically identical to existing insulin products in terms of their amino acid configuration). Such insulin biosimilars require extensive testing and have a complex approval process, which can result in these agents costing significantly more than generic preparations. Recently, abasaglar insulin, a biosimilar to insulin glargine, has been given approval in Europe. Furthermore, newer, highly concentrated insulin formulations have been developed for patients on high insulin doses. ${ }^{22}$

\section{Potential new therapies in diabetes}

\section{Inhaled and oral insulin}

Inhaled insulin (Exubera ${ }^{\circledR}$, Pfizer Ltd) was available for a brief period in the mid 2000s but was taken off the market for commercial reasons. ${ }^{23}$ More recently, a new formulation of inhaled insulin (Technosphere ${ }^{\circledR}$, Mannkind Corp) was launched in the USA. It is a rapid-acting insulin, taken before meals, and appears effective in lowering glucose levels in patients with T1D and T2D. ${ }^{24}$ A cough seems to be a minor adverse effect but longer term effects on the respiratory system have not been excluded.

Oral delivery of insulin has been sought for many years. Delivery of insulin to the gastrointestinal tract may, in fact, have significant physiological advantages over subcutaneous or other routes, as insulin is delivered into the portal tract where it can exert optimum physiological action. Buccal and oral delivery systems have remained challenging because of inconsistency of absorption but protein encapsulation into nanoparticles has been suggested as having potential. ${ }^{25}$ As yet, no clinically or commercially viable drug has been developed.

\section{Implantable GLP-1 analogue and once weekly DPP-4 inhibitor}

An implantable GLP-1 analogue device has been developed that can deliver the GLP-1 analogue exenatide for a period of up to 12 months using an osmotic mini-pump device. ${ }^{26}$ This has been trialled in patients with poor glucose control and shown a sustained reduction in glycated haemoglobin over that period, with few adverse effects. Omarigliptin (Merck \& Co) is a once weekly DPP-4 inhibitor that has shown efficacy in patients with T2D. ${ }^{27}$

\section{Glucagon receptor antagonists}

Glucagon antagonises insulin and increases hepatic glucose output. Inhibition of the glucagon receptor using small-molecule ligands may be a promising method to improve glucose and clinical data using small-molecule antagonists is emerging. ${ }^{28}$

\section{Adiponectin receptor agonists}

Adiponectin is produced by adipocytes and appears to have important insulin sensitising and anti-proliferative effects. Low levels of circulating adiponectin can increase the risk of metabolic syndrome. ${ }^{29}$ An orally active adiponectin receptor agonist, AdipoRon, has been developed and shows some promise in pre-clinical studies. ${ }^{30}$

\section{Selective PPAR- $\gamma$ agonist}

Adverse effects of PPAR- $\gamma$ agonists, such a pioglitazone, include fluid retention and heart failure, and bone fracture. More selective PPAR- $\gamma$ agonists may not have such adverse effects. Phase III studies of balaglitazone suggest that it may have lower propensity for such side effects, although these data have not been substantiated as yet in large studies. ${ }^{31}$

\section{Fibroblast growth factor-21 mimetics}

Levels of fibroblast growth factor-21 (FGF-21) progressively increase in patients with impaired glucose tolerance to overt T2D to micro- or macrovascular complications. ${ }^{32}$ FGF-21 mimetics have shown beneficial metabolic effects in in vitro studies and clinical studies are underway.

\section{Case studies}

\section{Case study 1}

A 45-year-old South Asian man with T2D of 4 years duration is seen for review. His cardiovascular risk factors are well controlled. He tries hard with his diet, but admits he is sedentary because of his job as a taxi driver. His body mass index (BMI) is $27 \mathrm{~kg} / \mathrm{m}^{2}$ and latest glycated haemoglobin is $68 \mathrm{mmol} / \mathrm{mol}(8.5 \%)$. He is asymptomatic. His current glycaemic therapy is metformin $1,000 \mathrm{mg}$ twice daily.

As he is young, has no other comorbidities and a short duration of diabetes, glycaemic control should be tightened. A reasonable target according to NICE would be $53 \mathrm{mmol} / \mathrm{mol}$ (7.0\%). He is overweight and as a taxi driver needs to avoid hypoglycaemia assiduously, hence the addition of a gliptin, such as sitagliptin $100 \mathrm{mg}$ daily, would probably be appropriate, along with reinforcement of lifestyle measures.

\section{Case study 2}

A 56-year-old white European woman with T2D of 8 years duration is seen for review. Her cardiovascular risk factors are well controlled. She is on treatment for obstructive sleep apnoea (OSA). She is obese (BMI $33.2 \mathrm{~kg} / \mathrm{m}^{2}$ ) and struggles with her weight despite trying many diets. Her latest glycated 
haemoglobin is $74 \mathrm{mmol} / \mathrm{mol}(8.9 \%)$, treated with metformin $1,000 \mathrm{mg}$ twice daily, sitagliptin $100 \mathrm{mg}$ once daily and gliclazide $80 \mathrm{mg}$ twice daily. She feels tired but has no osmotic symptoms. She is keen to avoid therapy that may cause weight gain.

She is relatively young and glycaemic control should be improved. Insulin, pioglitazone or increased sulfonylurea therapy are all likely to cause weight gain. NICE guidance suggests GLP-1 therapy should be considered if BMI is above $35 \mathrm{~kg} / \mathrm{m}^{2}$. It does state, however, that if weight loss would improve obesity-related comorbidities (eg OSA), then GLP-1 therapy can be considered for patients with a BMI below $35 \mathrm{~kg} / \mathrm{m}^{2}$. Therefore, along with reinforcement of lifestyle change, replacing the gliptin with GLP-1 therapy should be considered to aid glycaemic control and weight loss.

\section{Case study 3}

A 76-year-old Jamaican man with T2D for 18 years duration is seen for review. He has a past history of a cerebrovascular accident and has chronic kidney disease (CKD) stage $3 b$ (eGFR $32 \mathrm{~mL} / \mathrm{min}$ ) and well controlled heart failure. His cardiovascular risk factors are well controlled. BMI is $25.5 \mathrm{~kg} / \mathrm{m}^{2}$. He currently takes metformin $1,000 \mathrm{mg}$ twice daily and gliclazide $160 \mathrm{mg}$ twice daily. Glycaemic control is poor, with a glycated haemoglobin level of $92 \mathrm{mmol} / \mathrm{mol}(10.6 \%)$. He complains of nocturia and tiredness.

Despite his age and comorbidities, this man is at risk of decompensation of his glucose control and is symptomatic. In addition, his metformin is likely to require cessation as his renal disease progresses. Pioglitazone is contraindicated because of heart failure. GLP-1 and SGLT-2 therapy are not options with this degree of renal impairment. Therefore, it is appropriate to consider insulin therapy, cessation of metformin and gliclazide, and possible introduction of linagliptin in view of his renal disease. The target for glycaemic control should not be set too low as he is at risk of hypoglycaemia. A target of $70 \mathrm{mmol} / \mathrm{mol}$ $(8.7 \%)$ would be reasonable.

\section{Case study 4}

A 34-year-old white European man with T2D is seen for review. He presented 2 years ago with marked hyperglycaemia and was put on to metformin 1,000 mg twice daily and gliclazide $160 \mathrm{mg}$ twice daily shortly after diagnosis. Despite this, his glycaemic control has not been adequate, with latest glycated haemoglobin of $70 \mathrm{mmol} / \mathrm{mol}(8.7 \%)$. He is asymptomatic and has been seeing a dietician regularly. BMI is $29 \mathrm{~kg} / \mathrm{m}^{2}$ but he has lost approximately $5 \%$ of body weight in the last 2 years, although weight has now plateaued. He is keen to lose more weight.

This man clearly needs improved glucose control. He does not fulfil criteria to commence a GLP-1 and insulin therapy or glitazone therapy is likely to lead to weight gain. Gliptins are unlikely to aid with weight loss, therefore, SGLT-2 therapy should be considered.

\section{Conclusions}

With a growth in prevalence of diabetes, newer therapies have become available to manage the condition. The primacy of managing lifestyle factors at all stages of diabetes cannot be overstated. In addition, while managing cardiovascular risk factors is important, managing glucose should not be neglected because people with poor glycaemic control can be asymptomatic and at risk of developing microvascular complications. While newer drugs are available, finding the combination that fits with a person's lifestyle, their likelihood of compliance and knowing when to adapt and change when the treatment fails are all important. Newer drug therapies are increasingly enabling clinicians to tailor therapies according to these and other factors. With an ever increasing burden of diabetes affecting many health systems worldwide, new therapies for preventing and managing diabetes and its complications will be required.

\section{Conflicts of interest}

The authors declare no conflicts of interest.

\section{References}

1 Diabetes UK. Diabetes in the UK in 2012: key statistics on diabetes. London: Diabetes UK, 2012. Available online at www.diabetes.org. uk/Documents/Reports/Diabetes-in-the-UK-2012.pdf [Accessed 27 June 2016].

2 National Institute for Health and Care Excellence. Type 2 diabetes in adults: management. NICE clinical guideline 28. London: NICE, 2015.

3 International Diabetes Federation. Managing older people with Type 2 diabetes. Global Guideline. Brussels: IDF, 2013.

4 Inzucchi SE, Bergenstal RM, Buse JB et al. Management of hyperglycemia in type 2 diabetes, 2015: a patient-centered approach: update to a position statement of the American Diabetes Association and the European Association for the Study of Diabetes. Diabetes Care 2015;38:140-9.

5 Health and Social Care Information Centre. National Diabetes Audit 2013-14 and 2014-15. Report 1: care processes and treatment targets. Leeds: HSCIC, 2016. Available online at www.hscic.gov.uk/ catalogue/PUB19900/nati-diab-rep1-audi-2013-15.pdf [Accessed 27 June 2016].

6 Viollet B, Guigas B, Sanz Garcia N et al. Cellular and molecular mechanisms of metformin: an overview. Clin Sci 2012;122:253-70.

7 Buse JB, DeFronzo RA, Rosenstock J et al. The Primary GlucoseLowering Effect of Metformin Resides in the Gut, Not the Circulation: Results From Short-term Pharmacokinetic and 12-Week Dose-Ranging Studies. Diabetes Care 2016;39:198-205.

8 Holman RR, Paul SK, Bethel MA, Matthews DR, Neil HAW. 10-year follow-up of intensive glucose control in type 2 diabetes. $N$ Engl J Med 2008;359:1577-89.

9 Wulffelé MG, Kooy A, Lehert P et al. Combination of insulin and metformin in the treatment of type 2 diabetes. Diabetes Care 2002;25:2133-40.

10 Chowdhury TA, Wright R, Yaqoob MM. Metformin in renal disease: pharmaceutical guidelines are too restrictive. BMJ 2015;350:h1758.

11 Monami M, Genovese S, Mannucci E. Cardiovascular safety of sulfonylureas: a meta-analysis of randomized clinical trials. Diabetes Obes Metab 2013;15:938-53.

12 Scott LJ. Repaglinide: a review of its use in type 2 diabetes mellitus. Drugs 2012;22:249-72.

13 Sari R, Balci MK, Akbas SH, Avci B. The effects of diet, sulfonylurea, and Repaglinide therapy on clinical and metabolic parameters in type 2 diabetic patients during Ramadan. Endocr Res 2004;30:169-77.

14 Belfort R, Harrison SA, Brown K et al. A placebo-controlled trial of pioglitazone in subjects with nonalcoholic steatohepatitis. $N$ Engl J Med 2006;355:2297-307. 
15 Zhu ZN, Jiang YF, Ding T. Risk of fracture with thiazolidinediones: an updated meta-analysis of randomized clinical trials. Bone 2014;68:115-23.

16 Tuccori M, Filion KB, Yin $\mathrm{H}$ et al. Pioglitazone use and risk of bladder cancer: population based cohort study. BMJ 2016;352:i1541.

17 Montori VM. The safety of incretin based drugs. BMJ. 2014;348:g2779.

18 Zinman B, Wanner C, Lachin JM et al. Empagliflozin, cardiovascular outcomes, and mortality in type 2 diabetes. $N$ Engl J Med 2015;373:2117-28.

19 Bloomgarden ZT. Sodium-glucose cotransporter 2 inhibitors and diabetic ketoacidosis. J Diabetes 2015;8:175-6.

20 Holst JJ. The physiology of glucagon-like peptide. Physiol Rev 2007;87:1409-39.

21 Chowdhury TA, Wright R, Charlton M. Insulin for the unititated. Clin Med 2014;14:623-9.

22 Dashora, U. Castro, E. Insulin U100, 200, 300 or 500? Br J Diab 2016;16:10-13.

23 Bailey CJ, Barnett AH. Why is Exubera being withdrawn? BMJ 2007;333:1156.

24 Rosenstock J, Lorber DL, Gnudi L et al. Prandial inhaled insulin plus basal insulin glargine versus twice daily biaspart insulin for type 2 diabetes: a multicentre randomised trial. Lancet 2010;375:2244-53.

25 Fonte P, Araújo F, Reis S, Sarmento B. Oral insulin delivery: how far are we? J Diabetes Sci Technol 2013;7:520-31.
26 Meier JJ, Nauck MA. Incretin-based therapies: where will we be 50 years from now? Diabetologia 2015;58:1745-50.

27 Sheu WH, Gantz I, Chen M et al. Safety and Efficacy of Omarigliptin (MK-3102), a novel once-weekly DPP-4 inhibitor for the treatment of patients with Type 2 Diabetes. Diabetes Care 2015;38:2106-14.

28 Hasegawa F, Niidome K, Migihashi C et al. Discovery of furan2-carbohydrazides as orally active glucagon receptor antagonists. Bioorg Med Chem Lett 2014;24:4266-70.

29 Caselli C. Role of adiponectin system in insulin resistance. Mol Genet Metab 2014;113:155-60.

30 Okada-Iwabu M, Yamauchi T, Iwabu M et al. A small-molecule AdipoR agonist for type 2 diabetes and short life in obesity. Nature 2013;503:493-9.

31 Henriksen K, Byrjalsen I, Qvist P et al. Efficacy and safety of the PPAR partial agonist balaglitazone compared with pioglitazone and placebo: a phase III, randomized, parallel-group study in patients with type 2 diabetes on stable insulin therapy. Diabetes Metab Res Rev 2011;27:392-401.

32 Liu JJ, Foo JP, Liu S, Lim SC. The role of fibroblast growth factor 21 in diabetes and its complications: A review from clinical perspective. Diabetes Res Clin Pract 2015;108:382-9.

Address for correspondence: Dr T A Chowdhury, Department of Diabetes and Metabolism, 7th Floor, John Harrison House, The Royal London Hospital, Whitechapel, London E1 1BB, UK. Email:Tahseen.Chowdhury@bartshealth.nhs.uk
'Smoking is the biggest avoidable cause of death and disability, and social inequality in health, in the UK.'

\section{Nicotine without smoke Tobacco harm reduction}

Since e-cigarettes became available in the UK in 2007, their use has been surrounded by medical and public controversy. This new 200-page report from the Royal College of Physicians examines the science, public policy, regulation and ethics surrounding e-cigarettes and other nontobacco sources of nicotine, and addresses these controversies and misunderstandings with conclusions based on the latest available evidence.

ISBN 978-1-86016-600-6 £15 including $p+p$ or free to download

\section{Royal College} of Physicians
Download the report: rcplondon.ac.uk/nicotine

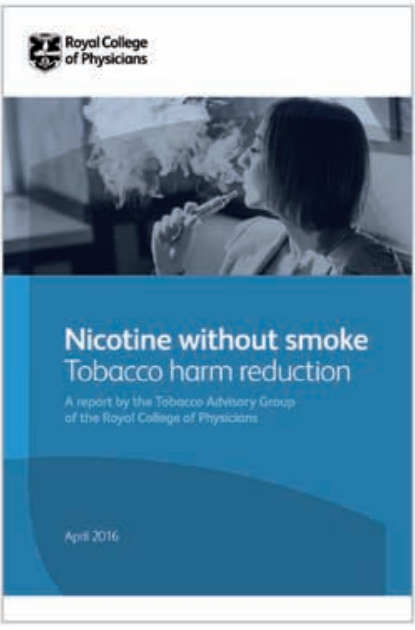

Order a copy: shop.rcplondon.ac.uk 